\title{
Probabilistically Shaped 4-PAM for Short-Reach IM/DD Links with a Peak Power Constraint
}

Wiegart, Thomas; Da Ros, Francesco; Yankov, Metodi Plamenov; Steiner, Fabian; Gaiarin, Simone; Wesel, Richard

Published in:

Journal of Lightwave Technology

Link to article, DOI:

10.1109/JLT.2020.3029371

Publication date:

2020

Document Version

Peer reviewed version

Link back to DTU Orbit

Citation (APA):

Wiegart, T., Da Ros, F., Yankov, M. P., Steiner, F., Gaiarin, S., \& Wesel, R. (2020). Probabilistically Shaped 4PAM for Short-Reach IM/DD Links with a Peak Power Constraint. Journal of Lightwave Technology, 39(2), 400405. https://doi.org/10.1109/JLT.2020.3029371

\section{General rights}

Copyright and moral rights for the publications made accessible in the public portal are retained by the authors and/or other copyright owners and it is a condition of accessing publications that users recognise and abide by the legal requirements associated with these rights.

- Users may download and print one copy of any publication from the public portal for the purpose of private study or research.

- You may not further distribute the material or use it for any profit-making activity or commercial gain

- You may freely distribute the URL identifying the publication in the public portal 


\title{
Probabilistically Shaped 4-PAM for Short-Reach IM/DD Links with a Peak Power Constraint
}

\author{
Thomas Wiegart, Student Member, IEEE, Francesco Da Ros, Senior Member, IEEE, Metodi Plamenov \\ Yankov, Member, IEEE, Fabian Steiner, Student Member, IEEE, Simone Gaiarin, Member, IEEE, \\ Richard D. Wesel, Senior Member, IEEE
}

\begin{abstract}
Probabilistic shaping for intensity modulation and direct detection (IM/DD) links is discussed and a peak power constraint determined by the limited modulation extinction ratio (ER) of optical modulators is introduced. The input distribution of 4-ary unipolar pulse amplitude modulation (PAM) symbols is optimized for short-reach transmission links without optical amplification nor in-line dispersion compensation. The resulting distribution is symmetric around its mean allowing to use probabilistic amplitude shaping (PAS) to generate symbols that are protected by forward error correction (FEC) and that have the optimal input distribution. The numerical analysis is confirmed experimentally for both an additive white Gaussian noise (AWGN) channel and a fiber channel, showing gains in transmission reach and transmission rate, as well as rate adaptability.
\end{abstract}

Index Terms-Probabilistic Shaping, 4-PAM, Intensity Modulation, Direct Detection, Peak Power Constraint

\section{INTRODUCTION}

Noncoherent transceivers based on intensity modulation (IM) and direct detection (DD) are currently the preferred choice for short reach links, e.g., data center interconnects or metro connections up to $100 \mathrm{~km}$. Here the key requirements are low power consumption, low complexity, low cost, and minimum latency [1]. As a result, short reach links focus on avoiding energy-hungry optical amplifiers and in-line optical dispersion compensation as these components would increase the latency and the power budget of the system.

Recently, probabilistic shaping (PS) and especially probabilistic amplitude shaping (PAS) [2], [3] received broad interest from academia and industry for coherent optical communications. PAS enables rate adaptation and a performance close to the Shannon limit. PAS is compatible with standard forward-error correction (FEC) schemes, but requires a symmetric input distribution to synthesize the optimal distribution after FEC encoding. Most works apply PS to an average power constrained additive white Gaussian (AWGN) channel with quadrature-amplitude modulation (QAM). Only recently, PS has started being investigated for IM/DD transmission, which imposes a non-negativity constraint on the channel input $X$,

T. Wiegart and F. Steiner are with the Institute for Communications Engineering, Technical University of Munich (TUM). E-Mail(s): \{thomas.wiegart, fabian.steiner\}@tum.de

F. Da Ros, M. P. Yankov, and S. Gaiarin are with DTU Fotonik, Technical University of Denmark (DTU). E-Mail(s): \{fdro, meya, simga $\} @$ fotonik.dtu.dk

R. Wesel is with the Communications System Laboratory, University of California Los Angeles (UCLA).

E-Mail: wesel@ucla.edu. as it is used to modulate the intensity of the electric field. In this case, an average (optical) power constraint is expressed as $\mathrm{E}[X][4]$, and the resulting optimal input distribution is asymmetric (e.g., [5]) so that PAS can not be employed directly.

In [5], the authors considered 4-ary pulse-amplitude modulation (PAM), optimized the input distribution and analyzed achievable rates for $56 \mathrm{~GB}$ aud transmission. This first experimental demonstration included both optical amplification and in-line dispersion compensation. Under these conditions, an average-power constraint was applied in the PS optimization, resulting in an exponential distribution that yields close to optimal performance. However, no coded results are provided. By using optical amplification, the system was not limited by the ER of the optical modulator, which normally limits the launch power in practical transmission scenarios, thus justifying the average-power constraint. PS for similar setups including optical amplifiers has recently also been considered in, e.g., [6], [7].

In [8] and [9], instead, the authors consider 4-PAM and 8PAM for un-amplified transmission. They propose to slightly modify the exponential distribution and assign the same probability to two symbols such that using PAS for FEC integration is possible. Improvements in receiver sensitivity of approximately $1 \mathrm{~dB}$ are observed. However, the additional modulator loss for the PS modulation format is not taken into account. As the authors point out in [8], operating the PS modulation format with the same ER as the uniform modulation format leads to a reduction in launch power by $1.5 \mathrm{~dB}$. Overall, the reduction of launch power is higher than the gain in receiver sensitivity and thus PS with an exponential-like distribution effectively shortens the reach. The reduced launch power for the exponential-like distribution optimized with an average power constraint can not be compensated in the absence of an optical amplifier.

In this work, we investigate PS for practical IM/DD links with no optical amplifiers nor optical dispersion compensation. Unlike previous works, we introduce a peak power constraint in the PS optimization. The peak-power constraint originates from the need to avoid increasing the optical loss in the Mach Zehnder modulator (MZM) due to its limited ER. The same peak-to-peak driving voltage is considered for both uniform and PS formats and similar optical loss are therefore targeted to avoid penalizing the PS modulation. This approach is even more relevant when alternative modulation schemes are considered, e.g., directly modulated lasers or electro-absorption 
modulated lasers [10].

The peak power constraint implies that the optimal distribution is discrete with a finite number of mass points [11]. The optimization of the achievable rate for symbol-metric decoding (SMD) and bit-metric decoding (BMD) shows that the optimal distribution is symmetric around $\mathrm{E}[X]$ and is fundamentally different from the ones suggested previously (e.g., [8]). We discuss potential gains using a linear AWGN model first and conduct optical back-to-back (B2B), i.e., AWGN channel, and realistic transmission experiments for 4-PAM transmission with symbol rates between 8 GBaud and 32 GBaud.

The remaining of the paper is organized as follows. In Section II the model of the extinction ratio constrained IM/DD system is introduced, and the PS optimization is discussed showing achievable bit- and symbol- metric decoding performance. In Section III the setup used for the experimental validation is described and the measurement results are reported. In Section IV the main conclusions are drawn.

\section{SYSTEM MODEL}

The IM/DD link is modeled by

$$
Y=\left|\sqrt{|X|}+N_{\mathrm{opt}}\right|^{2}+N_{\mathrm{el}}
$$

where $N_{\text {opt }}$ and $N_{\text {el }}$ denote additive noise in the optical and electrical domain, respectively, and the random variable (RV) $X$ denoting the transmit symbol has alphabet

$$
\mathcal{X}=\{0,1,2,3\} .
$$

As $X$ is modulated onto the intensity (i.e., the power) of the optical signal, the optical amplitude is proportional to the square root of the absolute value of $X$. At the receiver, squarelaw detection is performed, i.e., one obtains an electrical current proportional to the squared absolute value of the impinging optical amplitude. Due to typically low transmit powers of IM/DD systems, the Kerr nonlinearity of the fiber was neglected. This model also does not capture the impact of chromatic dispersion (CD). We will discuss the validity of this model based on the experimental results in Sec. III.

In this paper, we consider un-amplified links and the signal laser has an optical SNR in excess of $45 \mathrm{~dB}$. The noise contributions are thus dominated by electrical noise sources, e.g., electrical transmitter and receiver amplifiers, digital-toanalog and analog-to-digital converter noise. By neglecting the optical noise contributions, the system model simplifies to a linear AWGN model of the form

$$
Y=|X|+N_{\mathrm{el}}
$$

where $N_{\mathrm{el}}$ is a Gaussian distributed $\mathrm{RV}$ with zero mean and variance $\sigma^{2}$. In order to ensure a fair comparison, we aim for the same ER for uniform and shaped signaling, i.e., the MZM is operated with the same peak-to-peak driving voltage for both modes. Thus we have a peak power constraint on the input signal $X$, which is implicitly included in the model by constraining $X$ to the set $\mathcal{X}$. We define the peak-signal-tonoise ratio (PSNR) in the electrical domain as

$$
\operatorname{PSNR}=\frac{\max \{\mathcal{X}\}^{2}}{\sigma^{2}}=\frac{3^{2}}{\sigma^{2}} \text {. }
$$

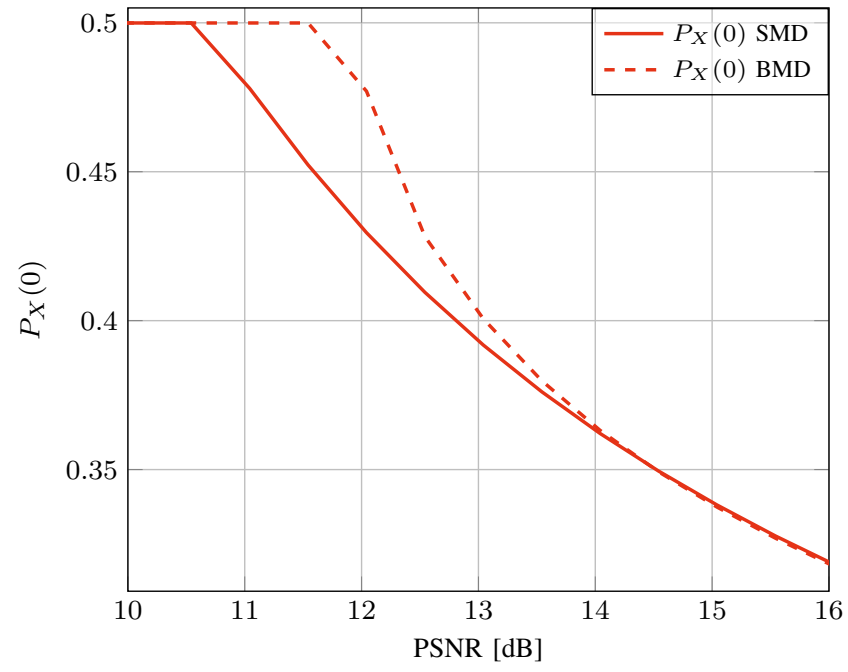

Fig. 1. Optimal $P_{X}(0)$ for SMD and BMD. If $P_{X}(0)=0.5$, this corresponds to OOK signalling, whereas $P_{X}(0)<0.5$ is 4 -PAM signalling.

Depending on the FEC architecture, either SMD or BMD metrics are relevant [12]. For BMD we label each constellation point $x \in \mathcal{X}$ with a two bit binary label, $x \mapsto \boldsymbol{b}=\left(b_{1} b_{2}\right)$. A binary reflected Gray code [13] performs well. To find the optimal distribution for PS, we solve the problem

$$
\max _{P_{X}} \quad R_{\mathrm{SMD} / \mathrm{BMD}} \quad \text { subject to } \quad X \in \mathcal{X}
$$

where

$$
R_{\mathrm{SMD}}=\mathrm{I}(X ; Y)=\mathrm{H}(X)-\mathrm{H}(X \mid Y)
$$

and

$$
R_{\mathrm{BMD}}=\left[\mathrm{H}(X)-\sum_{k=1}^{2} \mathrm{H}\left(B_{k} \mid Y\right)\right]^{+}
$$

with $[\cdot]^{+}=\max (0, \cdot)$ and where $\mathrm{I}(\cdot ; \cdot)$ denotes mutual information (MI), $\mathrm{H}(\cdot)$ denotes entropy and $\mathrm{H}(\cdot \cdot \cdot)$ denotes conditional entropy. The constraint $x \in \mathcal{X}$ is equivalent to a peak power constraint and a uniform spacing constraint on the signaling points. Using a similar approach as in [14, Proposition 2.3] and exploiting the fact that the mutual information is invariant with regard to a shift in the input constellation, it can be shown that the optimal distribution is symmetric around its mean $\mathrm{E}[X]$. Intuitively, this is the case because the input $X$ has an amplitude constraint on both sides (due to the peak power constraint and the non-negativity constraint) and the channel is linear. For 4-PAM and fixed mass point locations in $\mathcal{X}$, the optimization (5) boils down to a linesearch over a single parameter, namely the probability $P_{X}(0) \in[0,0.5]$, as $P_{X}(3)=P_{X}(0)$ and $P_{X}(1)=P_{X}(2)=\left(1-2 \cdot P_{X}(0)\right) / 2$.

The optimal $P_{X}(0)$ is shown in Fig. 1 for SMD and BMD. The plot also indicates the optimal constellation cardinality for each noise power. For low noise powers, $P_{X}(0)=0.5$ indicates that the optimal signaling uses a constellation with two mass points only, i.e., OOK. The optimal constellation cardinality depends on the decoding metric: for BMD, the operation range of OOK is larger than for SMD. With increasing PSNR the optimal distribution changes from 4-PAM with high 


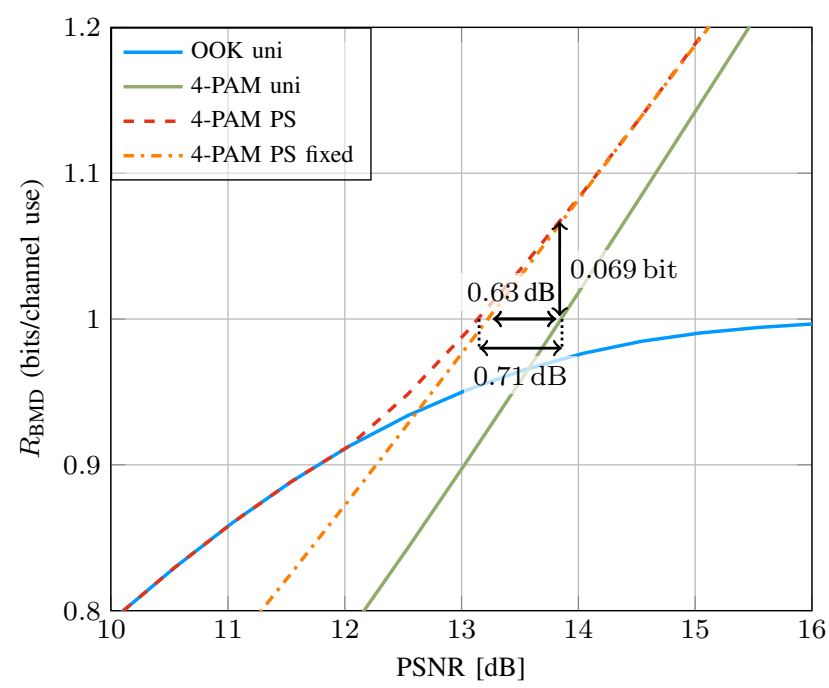

(a) Achievable rates with BMD.

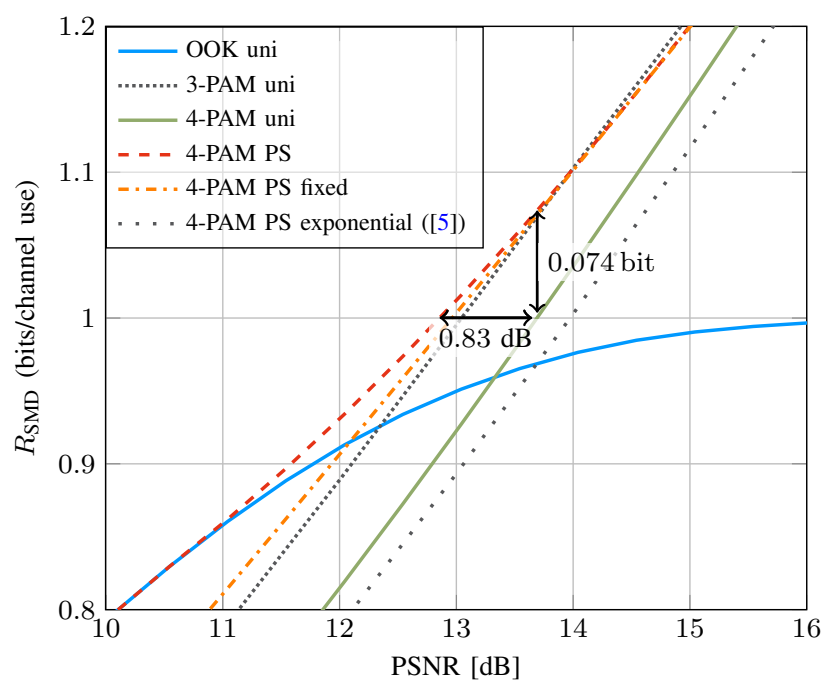

(b) Achievable rates with SMD.

Fig. 2. Achievable rates assuming the linear system model (3) and using BMD (a) and SMD (b), respectively. The distribution for the shaped curve (4-PAM PS) is chosen according to Fig. 1 .

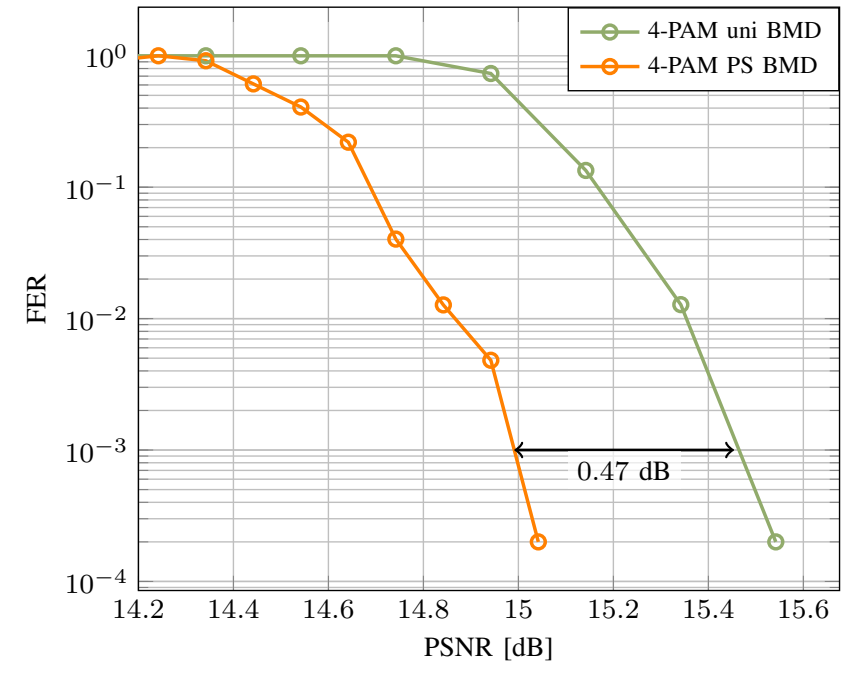

Fig. 3. Coded results for 4-PAM at a spectral efficiency of $1 \mathrm{bpcu}$ assuming the linear system model.

probability mass at the outer two points towards uniform 4PAM.

The corresponding achievable rates for uniform ("4-PAM uni" in the legend) and optimized PS signaling ("4-PAM PS") are shown in Figs. $2 \mathrm{a}$ and $2 \mathrm{~b}$ for BMD and SMD, respectively. We observe a gain of $0.83 \mathrm{~dB}$ in SNR for PS with SMD at 1.0 bits/channel use (bpcu). For BMD, the gain is $0.71 \mathrm{~dB}$. We also depict the achievable rates for the distribution $P_{X}=(0.35,0.15,0.15,0.35)$ ("4-PAM PS fixed") which will be used in the optical experiment of Sec. III. As can be inferred from Fig. 2, this $P_{X}$ performs close to the optimal distribution for a wide range of operating points. Here, the gain for BMD is $0.63 \mathrm{~dB}$ at a rate of $1.0 \mathrm{bpcu}$. Compared to the bipolar setting (see [2, Table 3]), BMD with PS exhibits a non-negligible gap to SMD for the unipolar case. The gap between SMD and BMD can be closed by using a non-binary FEC code or using a binary FEC code with multi-level coding and multistage decoding [15].

As the optimal input distribution $P_{X}$ is symmetric around $\mathrm{E}[X]$, we can use PAS with FEC. This is in contrast to previous works [5], [8] where an average power constraint is considered and the best distribution is asymmetric.

The exponential distribution from [5], optimized for an average power constraint, is also shown in Fig. $2 \mathrm{~b}$ as reference ("4-PAM PS exponential"). In the depicted range of Fig. 2b, the achievable rate for such a distribution under a realistic peak power constraint is even lower than in the uniform case.

We validate the information theoretic results of Fig. 2 through finite length simulations with low-density parity-check (LDPC) codes in Fig. 3. The LDPC code is regular and has degree 3 variable nodes (VNs) and a blocklength of around $10^{4}$ bits. The code was constructed using a protograph based design [16] with girth optimization techniques ensuring a girth of at least six. The uniform scenario uses a rate $1 / 2$ code $(100 \% \mathrm{OH})$, while the PS scenario uses a rate $0.56(78 \%$ $\mathrm{OH})$ code with PAS and constant composition distribution matching (CCDM) [17]. We use sum-product decoding with 50 iterations. At a frame error rate (FER) of $10^{-3}$, we observe a gain of $0.47 \mathrm{~dB}$. Tailored code designs, e.g., [18], can further improve the performance.

Although the achievable gains in terms of power efficiency are smaller in a peak-power limited non-coherent setting compared to average-power limited coherent transmission, we emphasize that probabilistic shaping can also be used to introduce rate adaptivity to the transmission system, by adjusting the shaping and FEC overheads. In particular, one can operate 4-PAM at PSNR levels where (uniform) 3-PAM (see black curve in Fig. 2b) would be used. By operating with 4-PAM instead of 3-PAM, an easier integration with binary FEC and binary input data is possible. 


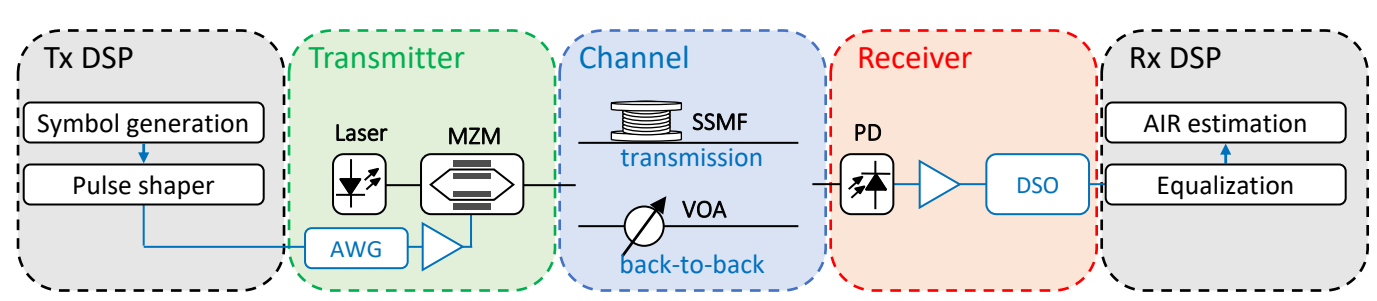

(a) Setup for back to back and transmission experiments.

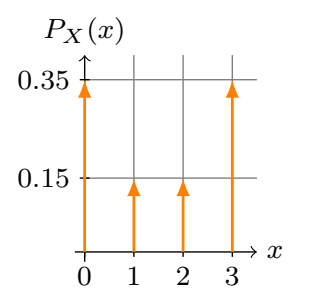

(b) Input distribution used for the experimental setup.

Fig. 4. Experimental setup and optimized input distribution.

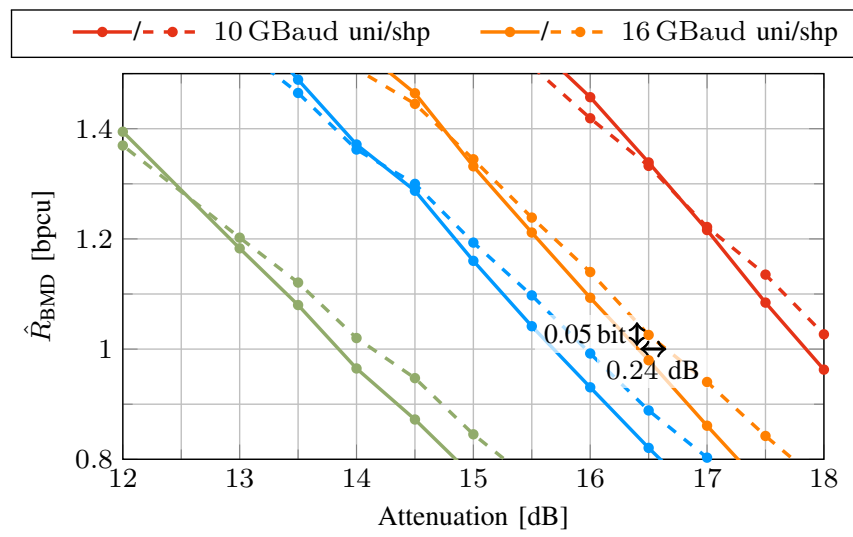

(a) $\hat{R}_{\mathrm{BMD}}$ estimates versus optical attenuation of the VOA.

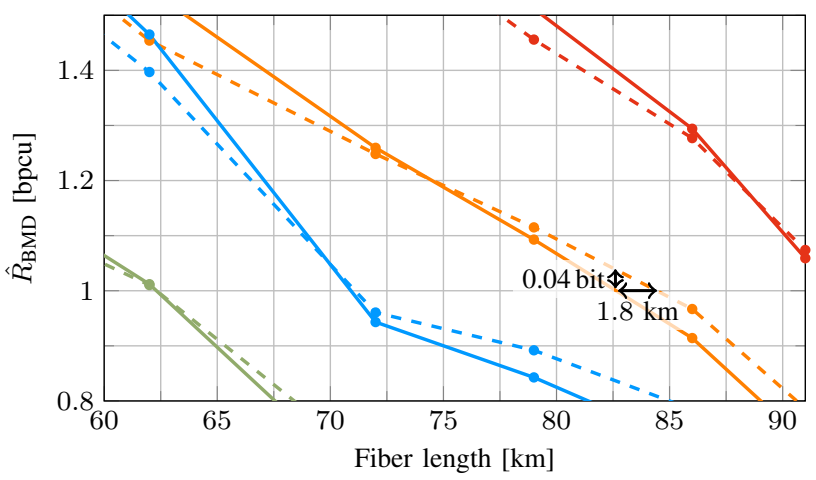

(c) $\hat{R}_{\mathrm{BMD}}$ estimates versus fiber length for transmission over SSMF.

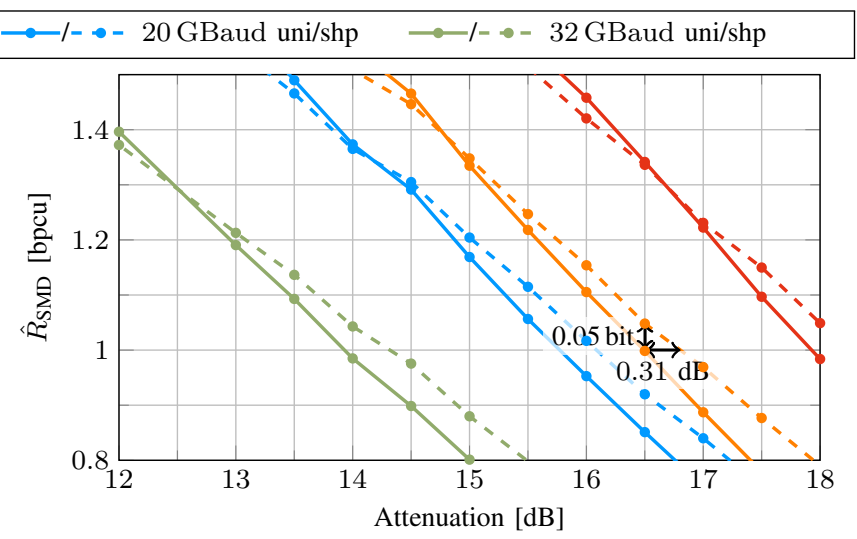

(b) $\hat{R}_{\mathrm{SMD}}$ estimates versus optical attenuation of the VOA.

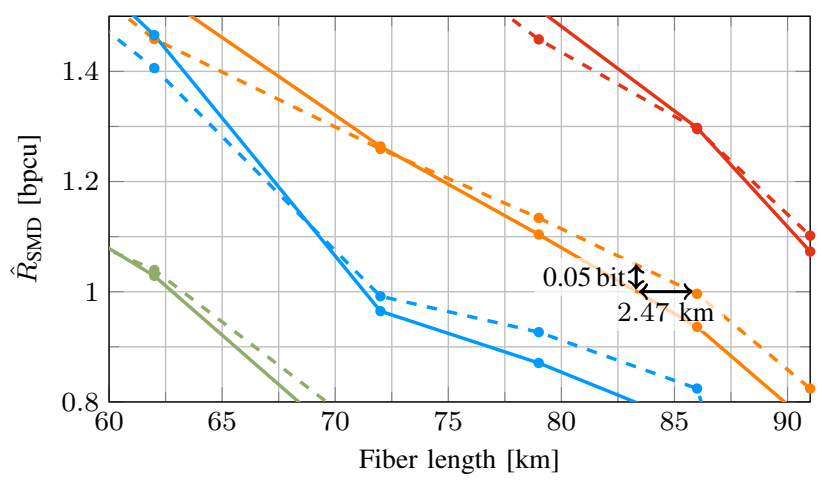

(d) $\hat{R}_{\mathrm{SMD}}$ estimates versus fiber length for transmission over SSMF.

Fig. 5. Experimental results for uniform and shaped (with the distribution depicted in Fig. 4b) transmission and different symbol rates: (a) and (b) depict $\hat{R}_{\mathrm{BMD}}$ and $\hat{R}_{\mathrm{SMD}}$ estimates with linear optical attenuation, respectively. and (c) and (d) depict $\hat{R}_{\mathrm{BMD}}$ and $\hat{R}_{\mathrm{SMD}}$ estimates for fiber transmission including dispersion effects, respectively.

\section{EXPERIMENTAL SETUP AND RESULTS}

The experimental setup is shown in Fig. 4a. Uniform or shaped (with the distribution shown in Fig. 4b) 4-PAM sequences are generated, rectangular pulse shaping is applied and the time domain waveforms are loaded into an arbitrary waveform generator (AWG, $64 \mathrm{GSa} / \mathrm{symbol}$ ). The AWG output is amplified and used to drive a MZM in order to encode the 4-PAM signal onto an optical carrier from an external cavity laser at $1550 \mathrm{~nm}$. The peak-to-peak driving voltage has been kept constant for all constellation shapes considered and was chosen to operate the MZM in the linear regime. Two scenarios have been investigated, a B2B configuration where the received power was varied via a variable optical attenuator (VOA) and a transmission scenario where different lengths of SSMF ( $\alpha=0.2 \mathrm{~dB} / \mathrm{km}, D=17 \mathrm{ps} / \mathrm{nm} \cdot \mathrm{km}$ ) were considered. The launch power into the SSMF was $2.5 \mathrm{dBm}$, a limitation imposed by the insertion loss of the MZM. The baudrate was varied between 8 GBaud and 32 GBaud. At the receiver, a $45 \mathrm{GHz}$ photodetector (PD) followed by a low-noise electrical amplifier converts the signal back to the electrical domain before analog-to-digital (A/D) conversion using a $80-\mathrm{GSa} / \mathrm{s}$ digital storage oscilloscope (DSO). Offline digital signal processing (DSP) is then applied, consisting of a linear feed forward equalizer (FFE, 5 taps for B2B, 11 taps for fiber transmission), to partially mitigate for the impact of chromatic dispersion and frequency limitations of electrical components, and achievable information rate (AIR) estimation [12, Sec. VII-G]. The estimated AIR with SMD 


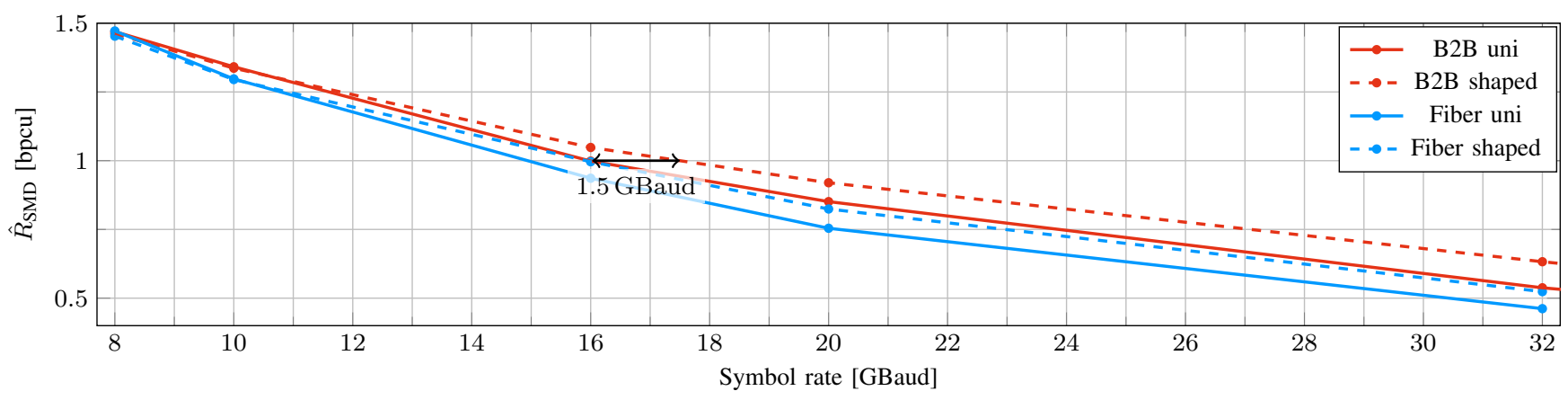

Fig. 6. $\hat{R}_{\mathrm{SMD}}$ estimates for uniform (solid curves) and shaped (dashed curves) transmission versus symbol rate for back-to-back measurements with optical attenuation of the VOA of $16.5 \mathrm{~dB}$ (red curves) and for fiber transmission with fiber length $L=86 \mathrm{~km}$ (blue curves).

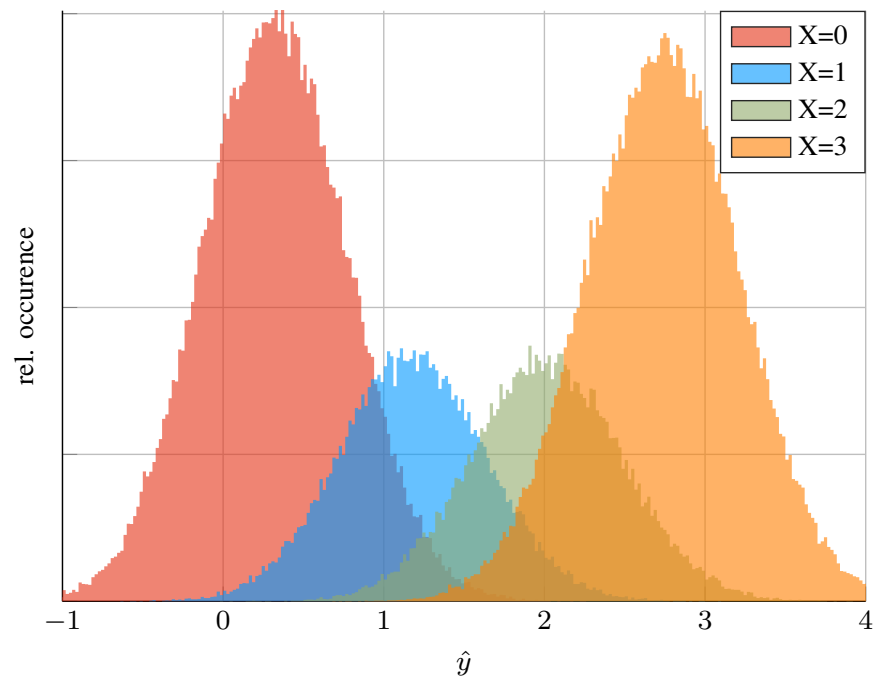

Fig. 7. Histogram of the estimated receive symbols $\hat{y}$ after the equalizer for transmit symbols $X \in \mathcal{X}$ with the distribution from Fig. 4b, transmitted with a symbol rate of 16 Gbaud over a fiber distance of $L=79 \mathrm{~km}$.

and BMD is denoted by $\hat{R}_{\mathrm{SMD}}$ and $\hat{R}_{\mathrm{BMD}}$, respectively.

From the definition of the AIR in (6) and (7) for SMD and BMD, respectively, it can be seen that the AIR contains both required overheads for shaping and forward error correction and thus provides a fair comparison between uniform and shaped signalling [12].

We depict the B2B results for BMD and SMD in Figs. 5a and $5 \mathrm{~b}$, respectively. The results considering this linear channel are in good agreement with the performance calculated for the channel model of (3) shown in Fig. 2. Considering a symbol rate of $16 \mathrm{GBaud}$ and for a target achievable rate of $1 \mathrm{bpcu}$, we observe that PS signaling allows an optical attenuation which is $0.24 \mathrm{~dB}$ and $0.31 \mathrm{~dB}$ higher than for the uniform case, for $\mathrm{BMD}$ and SMD, respectively. Alternatively, the rate gain is approximately $0.05 \mathrm{bpcu}(\approx 5 \%)$ and such a gain is consistent across the symbol rates investigated.

The transmission results over SSMF of different length are shown in Figs. 5c and 5d, again for BMD and SMD respectively. As the transmission length was varied by combining a different number of fiber spools of different lengths, the optical loss did not scale linearly with the transmission distance. Therefore, even though the CD scales linearly with distance, the rate decrease deviates from being monothonical with the increased fiber length. Nevertheless, for a symbol rate of $16 \mathrm{GBaud}$, we observe a rate gain of $0.05 \mathrm{bpcu}(\approx 5 \%)$, i.e., $0.8 \mathrm{Gbit} / \mathrm{s}$, compared to uniform signaling or a reach gain of up to $2.4 \mathrm{~km}(\approx 3 \%$ improvement) for SMD. The improvement slightly decreases down to $1.8 \mathrm{~km}$ for BMD. These results are still in line with those from the B2B setting. The key difference with the B2B results (Figs. 5a and 5b), is the impact of $\mathrm{CD}$ which becomes more significant as the symbol rate increases, reducing the improvement provided by shaping (at the a target achievable rate of $1 \mathrm{bpcu}$ ) for higher symbol rates. The impact of dispersion could be decreased by considering more complex equalization schemes which are beyond the scope of this work. More interesting, CD could be included within the channel model used for the shaping optimization. However, that would require to consider temporal effects within the shaping (i.e., shaping across several symbols), yielding a steep increase in complexity, as shown for coherent systems [19]. In the experiment, we observed that a slightly more uniform distribution shows better performance when the signal is affected by CD: the distribution depicted in Fig. 4b with $P_{X}(0)=0.35$ achieves slightly higher rates than the one optimized for the dispersion-less channel model of (3) $\left(P_{X}(0)=0.4\right.$ for a spectral efficiency of $\left.1 \mathrm{bpcu}\right)$.

Fig. 7 depicts the histogram of the estimated receive symbols $\hat{y}$ after the equalizer for transmission over $79 \mathrm{~km}$ of SSMF with a symbol rate of 16 Gbaud. The transmit symbols were chosen with the distribution in Fig. $4 \mathrm{~b}$ and we distinguish between the transmit symbols in $\mathcal{X}$ by different colors. Thus, Fig. 7 shows the empirical observation of the end-to-end channel law $p_{Y \mid X}$. This confirms the validity of the assumed linear channel model (3) with additive Gaussian noise.

Finally, Fig. 6 shows the rate achievable with SMD as a function of the transmitter symbol rate for a fiber distance of $86 \mathrm{~km}$ and for an optical attenuation of $16.5 \mathrm{~dB}$. At the target rate of $1 \mathrm{bpcu}$, both $\mathrm{B} 2 \mathrm{~B}$ and fiber channel lead to an increase in transmission rate of approx. 1.5 GBaud.

\section{CONCLUSION}

We investigated PS of 4-PAM for short reach links using IM/DD. In contrast to previous works, we investigated a 
more realistic short reach transmission scenario without optical amplification nor in-line dispersion compensation. More importantly, our work considers for the first time that the modulated signal is limited by the finite ER of the modulator and thus the peak power of the input symbols is constrained. Interestingly, the optimal input distribution with a peak power constraint has a symmetry and we can directly use PAS. We showed numerical gains of $0.47 \mathrm{~dB}$ using PAS and LDPC codes. The numerical analysis is validated through optical experiments both for B2B (AWGN channel) and fiber transmission. For a spectral efficiency of $1 \mathrm{bpcu}$ with symbol rates between $8 \mathrm{GBaud}$ and $32 \mathrm{GBaud}$, we show a reach gain of $2.9 \%$ compared to uniform signalling, or, alternatively, a transmission rate gain of 1.5 GBaud. While the gains for $\mathrm{B} 2 \mathrm{~B}$ are consistent across the symbol rate range considered, for the fiber channel, the impact of dispersion decreases the shaping gain at higher symbol rates, as expected since CD is not accounted for in our simple channel model.

\section{ACKNOWLEDGEMENTS}

This work was supported by the DFG (grants KR 3517/9-1 and KR 3517/8-1), the ERC CoG FRECOM (grant n. 771878), the DNRF Research CoE SPOC (ref. DNRF123), the Villum Young Investigator program OPTIC-AI (grant n. 29344) and the National Science Foundation (NSF) grant CCF-1911166. Any opinions, findings, and conclusions or recommendations expressed in this material are those of the authors and do not necessarily reflect the views of the NSF.

\section{REFERENCES}

[1] K. Zhong, X. Zhou, J. Huo, C. Yu, C. Lu, and A. P. T. Lau, "Digital signal processing for short-reach optical communications: A review of current technologies and future trends," J. Lightw. Technol., vol. 36, no. 2, pp. 377-400, Jan 2018.

[2] G. Böcherer, F. Steiner, and P. Schulte, "Bandwidth efficient and rate-matched low-density parity-check coded modulation," IEEE Trans. Commun., vol. 63, no. 12, pp. 4651-4665, Dec. 2015.

[3] F. Buchali, F. Steiner, G. Böcherer, L. Schmalen, P. Schulte, and W. Idler, "Rate adaptation and reach increase by probabilistically shaped 64-QAM: An experimental demonstration," J. Lightw. Technol., vol. 34, no. 7, pp. 1599-1609, Apr. 2016.

[4] J. Kahn and J. Barry, "Wireless infrared communications," Proc. IEEE, vol. 85, no. 2, pp. 265-298, Feb./1997.

[5] T. A. Eriksson, M. Chagnon, F. Buchali, K. Schuh, S. ten Brink, and L. Schmalen, "56 Gbaud probabilistically shaped PAM8 for data center interconnects," in Proc. Eur. Conf. Optical Commun. (ECOC), Sep. 2017, pp. 1-3.

[6] H. He, Y. Chen, Q. Xiao, L. Zhou, and Z. Dong, "LDPC-coded probabilistic shaping PAM4 based on many-to-one mapping in WDMPON,” J. Lightw. Technol., pp. 1-1, 2020.

[7] J. Zhang, K. Wang, Y. Wei, L. Zhao, W. Zhou, J. Xiao, B. Liu, X. Xin, F. Zhao, Z. Dong, and J. Yu, " $280 \mathrm{~Gb} / \mathrm{s}$ IM/DD PS-PAM- 8 transmission over $10 \mathrm{~km}$ SSMF at O-band for optical interconnects," in Opt. Fiber Commun. Conf. (OFC) 2020. Optical Society of America, 2020

[8] Z. He, T. Bo, and H. Kim, "Probabilistically shaped coded modulation for IM/DD system," Optics Express, vol. 27, no. 9, pp. 12 126-12 136, Apr. 2019

[9] D. Kim, Z. He, T. Bo, Y. Yu, and H. Kim, "Transmission of 36-Gbaud PAM-8 signal in IM/DD system using pairwise-distributed probabilistic amplitude shaping," in Opt. Fiber Commun. Conf. (OFC) 2020. Optical Society of America, 2020.

[10] K. Zhang, Q. Zhuge, H. Xin, W. Hu, and D. V. Plant, "Performance comparison of DML, EML and MZM in dispersion-unmanaged short reach transmissions with digital signal processing," Optics Express, vol. 26, no. 26, pp. 34288-34304, Dec 2018.
[11] J. G. Smith, "The information capacity of amplitude- and varianceconstrained scalar Gaussian channels," Inf. and Control, vol. 18, no. 3, pp. 203-219, Apr. 1971.

[12] G. Böcherer, P. Schulte, and F. Steiner, "Probabilistic shaping and forward error correction for fiber-optic communication systems," $J$. Lightw. Technol., vol. 37, no. 2, pp. 230-244, Jan. 2019.

[13] F. Gray, "Pulse code communication," U. S. Patent 2 632 058, 1953.

[14] J. Huang and S. P. Meyn, "Characterization and computation of optimal distributions for channel coding," IEEE Trans. Inf. Theory, vol. 51, no. 7 , pp. 2336-2351, Jul. 2005.

[15] U. Wachsmann, R. F. Fischer, and J. B. Huber, "Multilevel codes: Theoretical concepts and practical design rules," IEEE Trans. Inf. Theory, vol. 45, no. 5, pp. 1361-1391, 1999.

[16] D. Divsalar, S. Dolinar, C. R. Jones, and K. Andrews, "Capacityapproaching protograph codes," IEEE J. Sel. Areas Commun., vol. 27, no. 6, pp. 876-888, Aug. 2009.

[17] P. Schulte and G. Böcherer, "Constant composition distribution matching," IEEE Trans. Inf. Theory, vol. 62, no. 1, pp. 430-434, Jan. 2016.

[18] F. Steiner, G. Böcherer, and G. Liva, "Protograph-based LDPC code design for shaped bit-metric decoding," IEEE J. Sel. Areas Commun., vol. 34, no. 2, pp. 397-407, Feb. 2016.

[19] M. P. Yankov, K. J. Larsen, and S. Forchhammer, "Temporal probabilistic shaping for mitigation of nonlinearities in optical fiber systems," $J$. Lightw. Technol., vol. 35, no. 10, pp. 1803-1810, 2017. 